\title{
Editorial
}

\section{Social Robotics}

\author{
Pier Giuseppe Rossi ${ }^{\text {a }}$
}

${ }^{a}$ University of Macerata, Italy,piergiuseppe.rossi@unimc.it

\begin{abstract}
Robotics is the current trend. Maybe there is too much emphasis on it. Moreover, the meanings associated with it are different, as shown by the meeting promoted by both Italian Society for Research on Media Education (SIREM) and Elearning, Media, Education and Moodlemot (EMEM) Italia 2016, as well as the more recent conference "Giocare a pensare. Metodi e tecnologie per l'uso didattico dei robot/Playing and thinking. Methods and technologies for the educational use of robots", organised by Edoardo Datteri and Luisa Zecca of the University of Milan-Bicocca in May 2017. The activities focussed on this topic are numerous, often fostered by the National Plan for Digital School, where robotics has a significant space. The proposals are "didactical scenarios built around educational robotics, logic and computational thinking, manual and digital artefacts, serious play and storytelling" in "the perspective of building transversal learning" (Plan for digital school, 50). The request is to introduce "the methodology of Problem Posing and Solving in the teaching of Maths (...) and elements of educational robotics in the curricula of high school" (ivi, 75). Finally the prescriptive recommendation: "all students should be offered paths on making educational robotics, Internet of Things" (ivi, 78). As we can see, robotics is being connected not only to problem-posing and inter-disciplinarity but also to the Internet of Things.

But, if we analyse the experiences being developed at schools, robotics is framed in different perspectives, and the goals of its introduction are not always clear. If we read the documents that describe the school activities in which robotics is mentioned, the wider-used sentence is "play and entertainment", while very rarely are references made to the goals and objectives of such activities: what are the competences that teachers are working on? General sentences such as "educational robotics lets teachers and students to work in a team to learn in a creative and fun way" are very frequent in schools' websites. But these statements are rarely motivated and the advantages/differences relative to other methodologies are rarely highlighted.
\end{abstract}

Such lack is due to the act of underestimating the relation between robotics and Internet of Things, i.e. the impact of robotics on the social and organisational aspects of our society is not always made clear, mostly in a future perspective. If robotics is useful because it is connected to coding, probably more sustainable and effective modalities could be found.

Robotics is not coding only: the robotic artefacts have bodies, have an impact on our body and on our sensorimotor modalities. The bee robot cannot just be programmed, but it moves and occupies the same space in which we move and has an impact on our perspective and on our orientation. It is simplistic to think about robotics as just for "learning by discovery; exploring problem-solving; recognising the positive role of the mistake", as we can read in the website of a well-known publisher.

Robotics is connected to the Internet of Things and emphasises the corporeity, often underestimated, but existing also in other artefacts in computer science. Now, with robots, the corporeity cannot be ignored anymore.

The Internet of Things is poised to increasingly change our behaviours in future. It enters into our homes, it simplifies some activities and satisfies, in a clever way, our needs and carries out acts. At the same time, it is structuring our actions and requires a change in the logic with which we live and inhabit the space and carry out daily actions. As specified by Paul Dumouchel and Luisa Damiano in "Vivre avec les robots" (2016), "the artificial agents that we created embody, and at the same time reflect, the relations of power, but also of support and solidarity that there are among us" (ivi, 320) and they remind us that social robotics involves creating technical objects, social agents that will be integrated in our social context, that force us to rethink affection as a continuous mechanism of inter-individual coordination (ibidem). These issues require the distancing from the approach of the classical cognitive sciences and of the first era of computer science, which had seen the computer as the primary metaphor to describe the functioning of our brain, and requires to embrace the approach of the "embodied mind" that places the intelligence of the body in the centre.

Developing from these needs, the need to comprehend as culture and society are being changed by the presence of robotics in our daily life and the need to analyse potential use of robots that interact with human beings, specifically with elderly people and people with autism spectrum disorder, a research field called social robotics has been 


\section{Social Robotics}

Rossi

developing in the past few years. This field analyses how robotics has an impact on our imagination, how our life and the way we think of it is being changed or could be changed, how it could be used in empathetic processes and interpersonal ones.

This REM issue wishes to offer a vision on this topic and faces the various aspects of social robotics from different perspectives. 